\title{
Inventory of Butterflies and Its Role to Promote Ecotourism in Northern Sindhupalchok District of Central Nepal
}

\author{
Bhaiya Khanal ${ }^{1}$ \\ ${ }^{1}$ Natural History Museum, Tribhuvan University, Swayambhu, Kathmandu, Nepal \\ baya2000@live.com
}

\begin{abstract}
Sindhupalchok is one of the largest districts of central Nepal with area occupancy of about $2542 \mathrm{~km}^{2}$. The northern side of this district exhibits complex physical feature where this study was carried out within the elevation of $850 \mathrm{~m}$ to $4300 \mathrm{~m}$. The changing elevation gradients along mountain habitats act to control the distribution of butterflies at its ascending range. This study made on different periods in 2009 and 2012 brought a list of 114 species of butterflies from three different destinations of the northern side that included areas like Melamchi, Timbu (central-northern), Helambu (northern) Nakote (north-western) Panch Pokhari and adjoining region (north-eastern). The diversity of butterflies declined gradually above 3500m towards Panch Pokhari whereas species richness was noticed high at 900-2700 m from Melamchi to Helambu route including Nakote, Shermathan and Tarkyghyang. Ecotourism promotional activity can also be linked to the butterfly watch that includes rare and attractive species carrying high interest to the visiting tourists to this region. These tourists intend to watch, document and photograph these butterflies besides birds and other wildlife species. The supplementary information on these insects including other faunal components can be helpful to provide impressive knowledge to the visiting tourists to this part.
\end{abstract}

Key words: complex, composition gradients, physiographic, species, tourists 


\section{INTRODUCTION}

This mid-mountain district has a rugged topography with changing altitudinal features from the low land to the snow-capped peak of the Jugal Himalaya (Department of Information (1971). This is one of the largest districts of Nepal with area coverage of $2542 \mathrm{~km}^{2}$ and stands at its geographical position of 27. 7666, 85.7.

The changing effect of gradients has controlled the distribution of butterflies representing lesser but interesting species at the upper zone. This feature has created differing ecosystem types where pristine habitats for many unique species of the flora and fauna are present (Pradhan, 2013).

Butterflies play significant role to sustain and smooth run of the ecosystem besides aesthetic values. This insect can contribute significantly to promote the ecotourism which can attract several nature tourists and researchers who desire to watch and photograph beautiful and rare butterflies in their natural settings. Mexico's monarch roosting area is benefitted from the tourism as butterfly enthusiasts travel around to photograph these little beauties (Ames, 2021).

Melamchi River is a tributary of the larger Indrawati River basin that originates from the Jugal Himal at an elevation of $5875 \mathrm{~m}$. It joins below to Indrawati River at the Melamchi valley floor. The length of the river is $41 \mathrm{~km}$ and widens at downstream to the south. The lower part of this district is mostly warm and covered with the riverine forest. This also contributed to the diversity richness of butterflies where different vegetation components are present in this warm forested part (Pradhan, 2012). Being enriched with highly valued biodiversity, interesting terrains and snow capped peaks, this area is still beyond the reach to prosperous tourism which also lack complete documentation of the valuable natural history existing here (Pradhan, 2013). The present study aims to depict some of the butterfly species occurred in study areas based on observation and to promote possible butterfly inclusive eco-tourism.

\section{Study area}

This study covered three different destinations which included a. Melamchi to Timbu, b. Ambathan to Helambu and c. Melamchi to Panchpokhari. Melamchi, Ambathan, and Timbu are known as the Intake Zone for the well known Melamchi Water Diversion Project (fig. 1). 
Melamchi: This is a narrow river valley located at its geographical position of 27.83, 85.56. The elevation of this place ranged from $800 \mathrm{~m}$ to above $1000 \mathrm{~m}$ where preferred habitats for many butterfly species can be found. General vegetation noted in this valley are Ageratum conizoides, Asparagus racemosus, Barleria cristata, Begonia picta, Cassia occidentalis, Commelina sp., Crotalaria albida, Euphorbia hirta, Koenigia sp., Osbeckia stellata etc (Polunin \& Stainton,1984).

Helambu: A popular tourist's destination has its geographical position of 28.0188, 85.5253 . It is located about $28 \mathrm{~km}$ to the northeast direction of Kathmandu city. General vegetation noticed here are Schima wallichii, Castanopsis indica, Tsuga dumosa Pinus roxburghii and Rhododendron. The upper part includes Alnus nepalensis, Quercus lamillosa, Quercus semicarpifolia etc. (Stainton, 1997).

Panchpokhari: A place with five lakes stands at its geographical position of 28.023 and 85.43 . The considered elevation for this study ranged from 1200-4300m. General vegetation en route and around this place include Rhododendron arboreum, Quercus semicarpifolia, Pinus wallichiana and Pinus roxburghii while alpine vegetation includes, Arundinaria sp, Rhododendron arboreum, Pinus wallichiana, Quercus semicarpifolia, Juniperus recurva, Betula sps etc (Polunin \& Stainton, 1984).

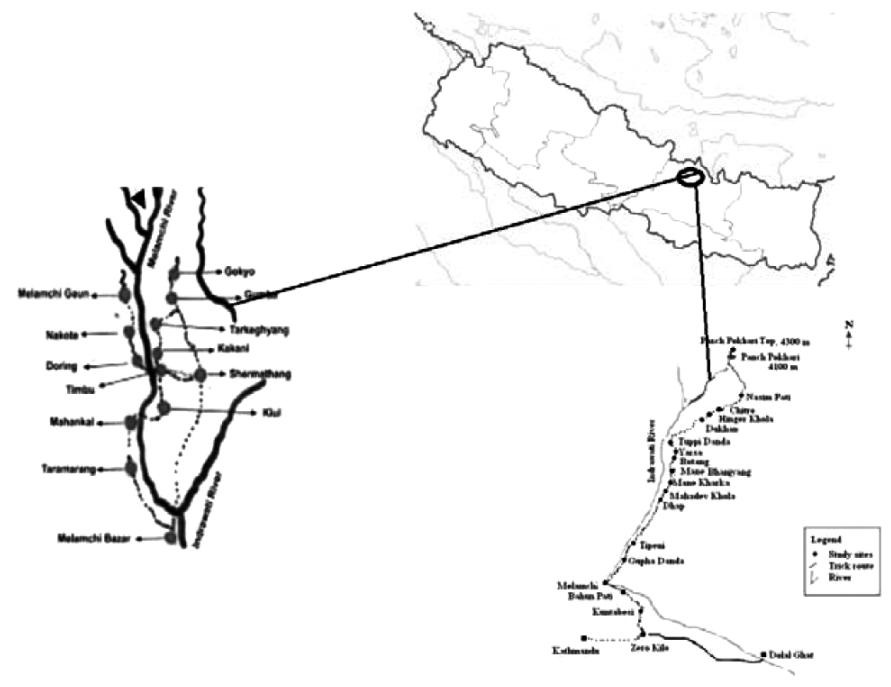

FIG.1. Study area at northern Sindhupalchok district depicting study routes at Melamchi, Helambu and Panchpokhari. 


\section{Climate}

Climatic pattern of Sindhupalchok distric is depicted in fig. 2. The valley floor of Melamchi is a warmer place influenced with tropical climate while subtropical climate occurs at the Timbu and Nakote areas. The temperate climate prevails at the Tarkyghyang and Shermathan and cold alpine climate can be experienced at the Panch Pokhari and adjoining areas.

The upper part of the Melamchi valley receives much rain than the floor area. The major precipitation occurs mainly due to the arrival of South-west monsoon in June to August. The average annual rainfall recorded in the Melamchi is about $2596 \mathrm{~mm}$. The winter rain is only for a brief period brought mainly by the western Mediterranean wind.

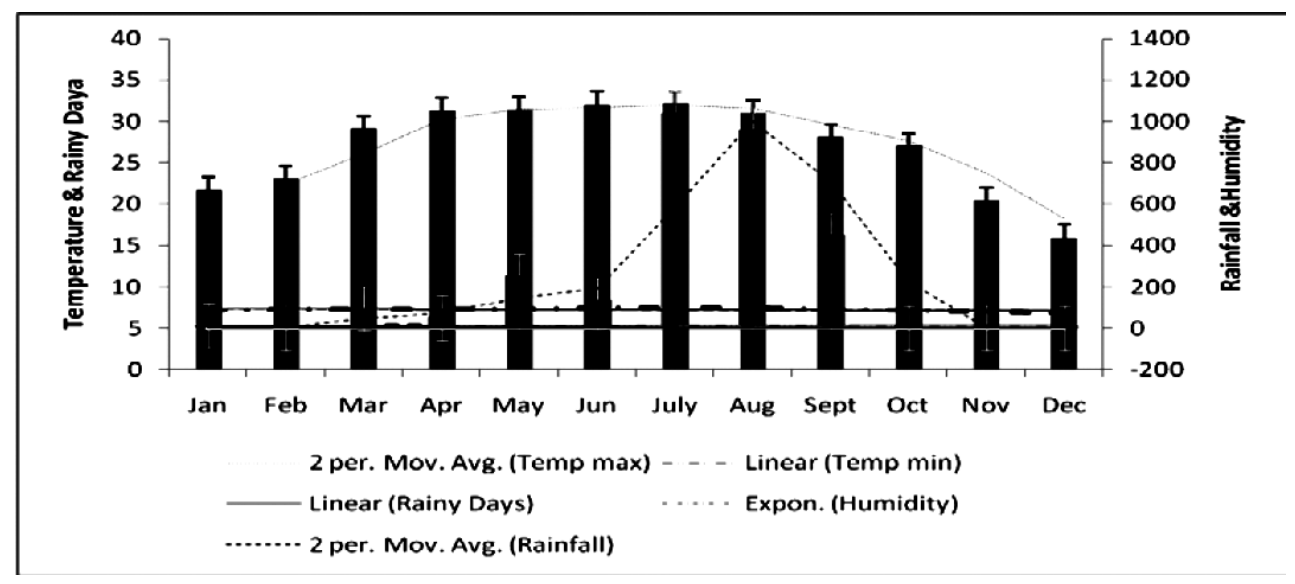

FIG. 2. Climatic pattern of Sindhupalchok district.

\section{MATERIALS AND METHODS}

Random field observation was made considering straight walk and side areas of the trail where different butterfly species were observed. Field identification was done with expert's knowledge and consulting books of Smith (1989) and Khanal \& Smith (1997).

\section{RESULTS AND DISCUSSION}

This work includes a total of 114 species which were recorded in 2009 and 2012 from a. Ambathan (1400m ) to Nakote (1940m), b. Melamchi Bazaar (850m) to Timbu (1350m), Tarkyghang (2754m), Shermathan (2520m) and c. Gupha DandaPanchpokhari (1200-4300m) of the northern side. । 
TABLE 1. Total number of species recorded in the study areas.

\begin{tabular}{|l|c|c|}
\hline \multicolumn{1}{|c|}{ Study location } & Elevation (m) & $\begin{array}{c}\text { Species record at each } \\
\text { location }\end{array}$ \\
\hline Melamchi Bazaar to Timbu & $859-1350$ & 79 \\
\hline $\begin{array}{l}\text { Helambu Area (Tarkyghyang, Shermathan } \\
\text { and Nakote) }\end{array}$ & $1940-2754$ & 83 \\
\hline Panchpokhari and adjoining area. & $1200-4300$ & 46 \\
\hline Total species record & \multicolumn{2}{|c|}{114} \\
\hline
\end{tabular}

The elevation range of $850-1350 \mathrm{~m}$ from Melamchi to Timbu brought a list of 79 species (table1). Troides aeacus, a CITES II listed species was seen at $850 \mathrm{~m}$ in the Melamchi, $1640 \mathrm{~m}$ and $1700 \mathrm{~m}$ in the Ambathan and Doring areas respectively (Annex 1). Among the recorded butterflies Catophaga lyncida, Spalgis epeus, Arophala eumolphus, Belenois aurota, Flos areste, Catacryshops strabo, Miletus chinensis, Lycaena phlaeas, Neptis soma, Neptis clinia, Cynitea lepidea, Stibochiona nicea, Polyuera athamas, Byasa alcinous, Sainia rhetenor, Cadugoides epycides, Danaus tytia, Tagiades menaka and Caltorius tulsi were assessed as locally rare species. They were sighted with less than three individuals in their frequencies.

The observation made at the Nakote, Tarkygyhang and Shermathang (1400-2754m) of the Helambu region revealed 83 species and the area from Gupha Danda (1200m) to the Panch Pokhari (4300m) represented 46 species where the diversity decreased with subsequent rise in elevation (Annex 1).

Among different activities essential to promote the ecotourism, butterfly watching is also a flourishing activity in many countries similar to bird watching and wildlife safaris. Eshun et al. (2014) mentioned how the ecotourism activities on the Bobiri Butterfly Sanctuary contribute to the conservation of the fauna and flora in the Sanctuary and towards the development of the local community. This study also found some semblance of ecotourism based on the Bobiri Butterfly Sanctuary as a conservation and development tool.

Helambu region has the basic infrastructure for tourism and has already been developed but reveals least information on the local biodiversity. Due to scenic beauty, incredible 
landscape, diverse cultural and natural diversity, Panchpokhari can be expected to attract many nature tourists if the knowledge of the high altitude biodiversity can be provided to them (Pradhan et al., 2009). Next interesting and possible destination could be the Bhairab Kund (4200m) which is a high land lake where unique butterflies and varied faunal components are sheltered in their pristine nature.

A list of the recorded species with their family, common and scientific names and location has been provided in the Annex 1 .

\section{ACKNOWLEDGEMENTS}

I am thankful to Professor Dr. Nirmala Pradhan, the Coordinator, for including me in this field research held in 2009 and 2012. Professor Dr. Mohan Siwakoti, the former Head of the Central Department of Botany, Tribhuwan University, is acknowledged for his help in plant identification during our visit to the Panch Pokhari in 2009. I would also like to thank to the Senior Botanist of NAST, Dr. Sandesh Bhattarai, for his field help to identify some butterfly related plants in 2012. Thanks are also due to Mr. Madan Krishna Shrestha, Mr. Vinod Thapa, Ms. Gita Thapa (Zoologists), Mr. Rajesh Tamang, Mr. Amin Pun, Ms. Suprava Shrestha and Ms. Sanam Prajapati (Botanists) for their essential help and support in the field.

\section{REFERENCES}

HAYLEY, A (2021) Why Are Butterflies Important? sciencing.com, https://sciencing. com/butterflies-important-8749269.html

DEPARTMENT OF INFORMATION(1971) Mechi Dekhi Mahakali. Madhyamanchal, Department of Information, Nepal Government. Kathmandu, Nepal.

ESHUN, G; FREMPONG, F; OSEI-WUSU ADJEI, P (2004) The Prospects of Ecotourism as a Conservation and Development Tool in Bobiri Butterfly Sanctuary in Ghana. Research on Humanities and Social Sciences, 4(22), ISSN (Paper) 2224-5766 ISSN (Online) 2225-0484 (Online).

KHANAL, B; SMITH, C (1995) Butterflies of Kathmandu valley. Tecpress Service, Bangkok, Thailand. 
PRADHAN, N (2013) Diversity And Status of Bryophytes in Panch Pokhari Region of The Northern Sindhupalchok District of Central Nepal. J. Nat. Hist. Mus. 27, 45-58.

PRADHAN, N.; KHANAL. B; SIWAKOTI, M; BHATTARAI, S; THAPA, V; THAPA, G (2009) An assessment of biodiversity in Panch Pokhari of Sindhupalchok District of Central Nepal Emphasizing Conservation Needs. Project Report, Rufford Small Grants Foundation, the United Kingdom (Unpublished).

PRADHAN, N (2012) An Approach to Determine the Diversity and Conservation Status of Bryophytes in Northern Sindhupalchok District of Nepal. Second $R S G$ report, Rufford Small Grant Foundation, UK (Unpublished).

POLUNIN, O; STAINTON, A (1984) Flowers of the Himalaya. Oxford University Press, Delhi, India.

SMITH, C (1989) Butterflies of Nepal. Tecpress Service, Bangkok, Thailand.

STAINTON, A (1997) Flowers of the Himalaya: A Supplement. Oxford University Press, India. 


\section{ANNEX 1}

\section{Recorded butterfly species at Melamchi, Helambu and Panchpokhari of the northern Sindhupalchok district of central Nepal.}

\begin{tabular}{|c|c|c|c|c|c|c|}
\hline S.N. & Family & Common Name & Scientific name & $\mathbf{M}$ & $\mathbf{H}$ & $\mathbf{P P}$ \\
\hline 1. & Pieridae & Large Cabbage White & $\begin{array}{l}\text { Pieris brassicae } \\
\text { Linnaeus }\end{array}$ & + & + & + \\
\hline 2. & & $\begin{array}{l}\text { Indian cabbage } \\
\text { White }\end{array}$ & $\begin{array}{l}\text { Pieris canidia } \\
\text { Sparman }\end{array}$ & + & + & + \\
\hline 3. & & $\begin{array}{l}\text { Common Grass } \\
\text { Yellow }\end{array}$ & $\begin{array}{l}\text { Eurema hecabe } \\
\text { Linnaeus }\end{array}$ & + & + & + \\
\hline 4. & & Spotless Grass Yellow & $\begin{array}{l}\text { Eurema laeta } \\
\text { Boisduval }\end{array}$ & + & + & + \\
\hline 5. & & Lemon Emigrant & $\begin{array}{l}\text { Catopsilia pomana } \\
\text { Fabricius }\end{array}$ & + & + & + \\
\hline 6. & & Mottled Emigrant & $\begin{array}{l}\text { Catopsilia pyranthe } \\
\text { Linnaeus }\end{array}$ & + & + & \\
\hline 7. & & Common Brimstone & $\begin{array}{l}\text { Gonepteryx rhamni } \\
\text { Linnaeus }\end{array}$ & + & + & + \\
\hline 8. & & $\begin{array}{l}\text { Dark Clouded } \\
\text { Yellow }\end{array}$ & $\begin{array}{l}\text { Colias fieldii } \\
\text { Menetries }\end{array}$ & + & + & + \\
\hline 9. & & $\begin{array}{l}\text { Pale Clouded } \\
\text { Yellow }\end{array}$ & Colias erate Esper & & + & + \\
\hline 10. & & Great Blackvein & $\begin{array}{l}\text { Metaporia agathon } \\
\text { Gray }\end{array}$ & + & & \\
\hline 11. & & Bath White & $\begin{array}{l}\text { Pontia daplidice } \\
\text { Linnaeus }\end{array}$ & + & & \\
\hline 12. & & Chocolate Albatross & $\begin{array}{l}\text { Catophaga lyncida } \\
\text { Cramer }\end{array}$ & + & + & \\
\hline 13. & & Psyche & $\begin{array}{l}\text { Cepora nerissa } \\
\text { Fabricius }\end{array}$ & + & & \\
\hline 14. & & Pioneer & $\begin{array}{l}\text { Belenois aurota } \\
\text { Fabricius }\end{array}$ & + & + & + \\
\hline
\end{tabular}




\begin{tabular}{|c|c|c|c|c|c|c|}
\hline 15. & & Psyche & $\begin{array}{l}\text { Leptosia nina } \\
\text { Fabricius }\end{array}$ & + & & \\
\hline 16. & & Hill Jezebel & $\begin{array}{l}\text { Delias belladonna } \\
\text { Fabricius }\end{array}$ & + & + & + \\
\hline 17. & & Redbase Jezebel & $\begin{array}{l}\text { Delias pasithoe } \\
\text { Linnaeus }\end{array}$ & + & & \\
\hline 18. & & Painted Jezebel & $\begin{array}{l}\text { Delias hyparete } \\
\text { Wallace }\end{array}$ & + & & \\
\hline 19. & & Yellow Orange Tip & Ixias pyrene Butler & + & + & \\
\hline 20. & Lycaenidae & Grass Jewel & $\begin{array}{l}\text { Freyeria trochilus } \\
\text { Freyer }\end{array}$ & + & + & \\
\hline 21. & & Apefly & $\begin{array}{l}\text { Spalgis epeus } \\
\text { Westwood }\end{array}$ & + & & \\
\hline 22. & & Bright Sunbeam & $\begin{array}{l}\text { Curetis bulis } \\
\text { Westwood }\end{array}$ & + & + & \\
\hline 23. & & Green Oakblue & $\begin{array}{l}\text { Arophala eumolphus } \\
\text { Cramer }\end{array}$ & + & & \\
\hline 24. & & Hooked Oakblue & $\begin{array}{l}\text { Arophala paramuta } \\
\text { DeNiceville }\end{array}$ & + & & \\
\hline 25. & & Plushblue & Flos areste Hewitson & + & & \\
\hline 26. & & Indian Oakblue & $\begin{array}{l}\text { Arophala atrax } \\
\text { Hewitson }\end{array}$ & + & + & \\
\hline 27. & & $\begin{array}{l}\text { Common } \\
\text { Hedgeblue }\end{array}$ & $\begin{array}{l}\text { Actyolepsis puspa } \\
\text { Horsfield }\end{array}$ & + & + & + \\
\hline 28. & & $\begin{array}{l}\text { Margined } \\
\text { Hedgeblue }\end{array}$ & $\begin{array}{l}\text { Celatoxia marginata } \\
\text { DeNiceville }\end{array}$ & + & & \\
\hline 29. & & Dark Cerulean & Jamides bochus Stoll & & + & + \\
\hline 30. & & Peablue & $\begin{array}{l}\text { Lampides boeticus } \\
\text { Linnaeus }\end{array}$ & + & + & + \\
\hline 31. & & Forget me not Blue & $\begin{array}{l}\text { Catacryshops strabo } \\
\text { Fabricius }\end{array}$ & & + & \\
\hline 32. & & Pale Hedgeblue & $\begin{array}{l}\text { Pseudozizeeria maha } \\
\text { Kollar }\end{array}$ & + & + & \\
\hline
\end{tabular}


52 Khanal: Inventory of Butterflies and Its Role to Promote Ecotourism.......

\begin{tabular}{|c|c|c|c|c|c|c|}
\hline 33. & & Common Brownie & $\begin{array}{l}\text { Miletus chinensis } \\
\text { Felder }\end{array}$ & + & & \\
\hline 34. & & $\begin{array}{l}\text { Longbanded } \\
\text { Silverline }\end{array}$ & $\begin{array}{l}\text { Spindasis lohita } \\
\text { Horsfield }\end{array}$ & + & & \\
\hline 35. & & Common Copper & $\begin{array}{l}\text { Lycaena phlaeas } \\
\text { Linnaeus }\end{array}$ & & + & + \\
\hline 36. & & Chapman's Cupid & $\begin{array}{l}\text { Everes argiades } \\
\text { Pallas }\end{array}$ & & + & \\
\hline 37. & & Pale Hedgeblue & Udara dilecta Moore & & & + \\
\hline 38. & & $\begin{array}{l}\text { Powdery Green } \\
\text { Sapphire }\end{array}$ & $\begin{array}{l}\text { Heliophorous tamu } \\
\text { Kollar }\end{array}$ & & + & \\
\hline 39. & & Purple Sapphire & $\begin{array}{l}\text { Heliophorus epicle } \\
\text { Godart }\end{array}$ & + & & \\
\hline 40. & & Green Sapphire & $\begin{array}{l}\text { Heliophorus androcles } \\
\text { Westwood }\end{array}$ & + & + & \\
\hline 41. & Nymphalidae & Peacock Pansy & $\begin{array}{l}\text { Precis almana } \\
\text { Linnaeus }\end{array}$ & & + & \\
\hline 42. & & Lemon Pansy & $\begin{array}{l}\text { Precis lemonias } \\
\text { Linnaeus }\end{array}$ & & + & \\
\hline 43. & & Chocolate Pansy & Precis iphita Cramer & & + & \\
\hline 44. & & Grey Pansy & Precis atlites Linnaeus & + & & \\
\hline 45. & & Lemon Pansy & $\begin{array}{l}\text { Precis orithya } \\
\text { Linnaeus }\end{array}$ & + & & \\
\hline 46. & & Red Admiral & Vanessa indica Herbst & & + & + \\
\hline 47. & & Painted Lady & $\begin{array}{l}\text { Vanessa cardui } \\
\text { Linnaeus }\end{array}$ & & + & + \\
\hline 48. & & Common Eggfly & $\begin{array}{l}\text { Hypolymnas bolina } \\
\text { Linnaeus }\end{array}$ & + & & \\
\hline 49. & & Yellow Sailor & Neptis ananta Moore & + & + & \\
\hline 50. & & Common Sailor & $\begin{array}{l}\text { Neptis hylas } \\
\text { Linnaeus }\end{array}$ & + & + & + \\
\hline 51. & & Pallas Sailor & Neptis sappho Pallas & + & & \\
\hline 52. & & Clear Sailor & Neptis clinia & & + & \\
\hline
\end{tabular}




\begin{tabular}{|c|c|c|c|c|c|}
\hline 53. & Sullied Sailor & Neptis soma Moore & & + & + \\
\hline 54. & Grey Count & $\begin{array}{l}\text { Cynitia lepidea } \\
\text { Butler }\end{array}$ & & + & \\
\hline 55. & Indian Fritillary & $\begin{array}{l}\text { Argyreus hyperbius } \\
\text { Linnaeus }\end{array}$ & + & + & + \\
\hline 56. & Common Nawab & $\begin{array}{l}\text { Polyura athamas } \\
\text { Drury }\end{array}$ & + & & \\
\hline 57. & Popinjay & Stibochiona nicea & + & & \\
\hline 58. & Common Laeopard & $\begin{array}{l}\text { Phalanta phalantha } \\
\text { Drury }\end{array}$ & + & + & + \\
\hline 59. & Common Lascar & Pantoporia hordonia & + & + & \\
\hline 60. & Indian Tortoiseshell & $\begin{array}{l}\text { Aglais cashmirensis } \\
\text { Kollar }\end{array}$ & + & + & + \\
\hline 61. & $\begin{array}{l}\text { Queen of Spain } \\
\text { Fritillary }\end{array}$ & $\begin{array}{l}\text { Issoria issaea } \\
\text { Doubleday }\end{array}$ & & + & + \\
\hline 62. & Common Baron & $\begin{array}{l}\text { Euthalia aconthea } \\
\text { Cramer }\end{array}$ & & + & + \\
\hline 63. & Sergeant Major & Abrota ganga Moore & & + & \\
\hline 64. & Common Sergeant & $\begin{array}{l}\text { Athyma perius } \\
\text { Linnaeus }\end{array}$ & + & + & \\
\hline 65. & Common Map & $\begin{array}{l}\text { Cyrestis thyodamus } \\
\text { Boisduval }\end{array}$ & + & + & + \\
\hline 66. & Orange Oakleaf & $\begin{array}{l}\text { Kallima inachus } \\
\text { Boisduval }\end{array}$ & & + & \\
\hline 67. & $\begin{array}{l}\text { Orange Staff } \\
\text { Sergeant }\end{array}$ & Athyma cama Moore & + & + & \\
\hline 68. & Himalayan Sergeant & $\begin{array}{l}\text { Athyma opalina } \\
\text { Kollar }\end{array}$ & + & + & + \\
\hline 69. & Circe & $\begin{array}{l}\text { Hestina nama } \\
\text { Doubleday }\end{array}$ & & + & \\
\hline 70. & Newar Threering & $\begin{array}{l}\text { Ypthima newara } \\
\text { Moore }\end{array}$ & + & + & + \\
\hline 71. & Himalayan Fivering & Ypthima sakra Moore & + & + & + \\
\hline
\end{tabular}


54 Khanal: Inventory of Butterflies and Its Role to Promote Ecotourism.......

\begin{tabular}{|c|c|c|c|c|c|}
\hline 72. & Treble Silverstripe & $\begin{array}{l}\text { Lethe baladeva } \\
\text { Moore }\end{array}$ & & & + \\
\hline 73. & $\begin{array}{l}\text { Common } \\
\text { Woodbrown }\end{array}$ & $\begin{array}{l}\text { Zophoessa sidonis } \\
\text { Hewitson }\end{array}$ & & + & + \\
\hline 74. & $\begin{array}{l}\text { Common } \\
\text { Bushbrown }\end{array}$ & $\begin{array}{l}\text { Mycalesis perseus } \\
\text { Fabricius }\end{array}$ & + & + & \\
\hline 75. & Brand Bushbrown & $\begin{array}{l}\text { Mycalesis mineus } \\
\text { Linnaeus }\end{array}$ & & + & \\
\hline 76. & Pallid Argus & $\begin{array}{l}\text { Callerebia scanda } \\
\text { Kollar }\end{array}$ & & + & + \\
\hline 77. & Ringed Argus & $\begin{array}{l}\text { Callerebia annada } \\
\text { Moore }\end{array}$ & & & + \\
\hline 78. & Striated Satyr & $\begin{array}{l}\text { Aulocera saraswati } \\
\text { Kollar }\end{array}$ & & + & \\
\hline 79. & Jungle Brown & $\begin{array}{l}\text { Orsotrioena medus } \\
\text { Fabricius }\end{array}$ & + & + & \\
\hline 80. & $\begin{array}{l}\text { Common Evening } \\
\text { Brown }\end{array}$ & $\begin{array}{l}\text { Melanitis leda } \\
\text { Linnaeus }\end{array}$ & + & + & \\
\hline 81. & $\begin{array}{l}\text { Common Indian } \\
\text { Crow }\end{array}$ & Euploea core Cramer & + & + & \\
\hline 82. & Striped Blue Tiger & $\begin{array}{l}\text { Euploea mulciber } \\
\text { Cramer }\end{array}$ & + & + & \\
\hline 83. & Glassy Tiger & Danaus aglea Stoll & + & + & \\
\hline 84. & Chestnut Tiger & Danaus tytia Oray & + & & + \\
\hline 85. & Plain Tiger & $\begin{array}{l}\text { Danaus chryssipus } \\
\text { Linnaeus }\end{array}$ & + & + & \\
\hline 86. & Common Tiger & $\begin{array}{l}\text { Danaus genutia } \\
\text { Cramer }\end{array}$ & + & + & + \\
\hline 87. & Blue Glassy Tiger & $\begin{array}{l}\text { Tirumala limniace } \\
\text { Cramer }\end{array}$ & + & & \\
\hline 88. & Yellow coster & $\begin{array}{l}\text { Acraea issoria } \\
\text { Hubner }\end{array}$ & + & + & + \\
\hline
\end{tabular}




\begin{tabular}{|c|c|c|c|c|c|c|}
\hline 89. & Riodinidae & Dark Judy & $\begin{array}{l}\text { Abisara fylla } \\
\text { Doubleday }\end{array}$ & + & + & \\
\hline 90. & & Punchinello & $\begin{array}{l}\text { Zemeros flegyas } \\
\text { Cramer }\end{array}$ & + & + & + \\
\hline 91. & Papilionidae & Great Mormon & $\begin{array}{l}\text { Papilio memnon } \\
\text { Linnaeus }\end{array}$ & + & & \\
\hline 92. & & Common Mormon & $\begin{array}{l}\text { Papilio polytes } \\
\text { Linnaeus }\end{array}$ & + & + & + \\
\hline 93. & & Spangle & $\begin{array}{l}\text { Sainia protenor } \\
\text { Cramer }\end{array}$ & + & + & \\
\hline 94. & & Common Rose & $\begin{array}{l}\text { Pachiliopta } \\
\text { aristolochae } \\
\text { Fabricius }\end{array}$ & + & & \\
\hline 95. & & Rose Windmill & $\begin{array}{l}\text { Atrophaneura } \\
\text { latrellei Donavan }\end{array}$ & + & + & + \\
\hline 96. & & Common Bluebottle & $\begin{array}{l}\text { Graphium sarpedon } \\
\text { Linnaeus }\end{array}$ & + & + & + \\
\hline 97. & & Red Helen & Menelaides helenus & + & + & \\
\hline 98. & & Common Peacock & $\begin{array}{l}\text { Achillides polyctor } \\
\text { Boisduval }\end{array}$ & + & + & + \\
\hline 99. & & Blue Peacock & $\begin{array}{l}\text { Achillides arcturus } \\
\text { Westwood }\end{array}$ & & + & + \\
\hline 100. & & Chinese Windmill & Byasa alcinous Klug & & + & + \\
\hline 101. & & Common Windmill & $\begin{array}{l}\text { Byasa polyeuctes } \\
\text { Doubleday }\end{array}$ & & + & + \\
\hline 102. & & Tailed Jay & $\begin{array}{l}\text { Idaides agamemnon } \\
\text { Linnaeus }\end{array}$ & + & + & \\
\hline 103. & & Common Redbreast & $\begin{array}{l}\text { Sainia rhetenor } \\
\text { Westwood }\end{array}$ & & + & \\
\hline 104. & & Lime Swallowtail & $\begin{array}{l}\text { Papilio demoleus } \\
\text { Linnaeus }\end{array}$ & + & & \\
\hline 105. & & Golden Birdwing & $\begin{array}{l}\text { Troides aeacus } \mathrm{C} \& \mathrm{R} \\
\text { Felder }\end{array}$ & + & + & \\
\hline
\end{tabular}




\begin{tabular}{|c|l|l|l|l|l|l|}
\hline 106. & & Lesser Mime & $\begin{array}{l}\text { Cadugoides epycides } \\
\text { Hewitson }\end{array}$ & + & & \\
\hline 107. & Hesperiidae & Fulvous Pied Flat & $\begin{array}{l}\text { Pseudocoladenia dan } \\
\text { Fabricius }\end{array}$ & + & + & + \\
\hline 108. & Grass Demon & $\begin{array}{l}\text { Udaspes folus } \\
\text { Cramer }\end{array}$ & + \\
\hline 109. & Restricted Demon & $\begin{array}{l}\text { Notocrypta } \\
\text { curvifascia } \text { Felder }\end{array}$ & + & + & + & + \\
\hline 110. & & Spotted Snowflat & $\begin{array}{l}\text { Mogiades menaka } \\
\text { Moore }\end{array}$ & $+\begin{array}{l}\text { Tagiades litigiosa } \\
\text { Moschler }\end{array}$ & + & + \\
\hline 111. & Water Snowflat & Paltorius tulsi & & + & + \\
\hline 112. & & $\begin{array}{l}\text { Small Branded } \\
\text { Swift }\end{array}$ & $\begin{array}{l}\text { Pelopidas mathias } \\
\text { Fabricius }\end{array}$ & & + \\
\hline 113. & & $\begin{array}{l}\text { Chinese Branded } \\
\text { Swift }\end{array}$ & $\begin{array}{l}\text { Pelopidas sinensis } \\
\text { Mabille }\end{array}$ & & + \\
\hline 114. & & & &
\end{tabular}

$\mathrm{M}=$ Melamchi (859-1350 m), $\mathrm{H}=$ Helambu area (Tarkyghyang, Shermathan and Nakote, 1940-2754 m), PP = Panch pokhari region (1200-4300), + = Presence. 